Proyecciones Journal of Mathematics

Vol. 30, No 3, pp. 319-327, December 2011.

Universidad Católica del Norte

Antofagasta - Chile

\title{
Accretive operators and Banach Alaoglu theorem in Linear 2-normed spaces
}

\author{
P. K. HARIKRISHNAN \\ MANIPAL INSTITUTE OF TECHNOLOGY, KARNATAKA \\ BERNARDO LA FUERZA GUILLÉN \\ UNIVERSIDAD DE ALMERÍA, SPAIN \\ and \\ K. T. RAVINDRAN \\ PAYYANUR COLLEGE, INDIA \\ Received : February 2011. Accepted : August 2011
}

\begin{abstract}
In this paper we introduce the concept of accretive operator in linear 2-normed spaces, focusing on the relationships and the various aspects of accretive, m-accretive and maximal accretive operators. We prove the analogous of Banach-Alaoglu theorem in linear 2- normed spaces, obtaining an equivalent definition for accretive operators in linear 2-normed spaces.
\end{abstract}

Mathematics Subject Classification : 41A65, $41 A 15$.

Keywords : Linear 2-normed spaces, sequentially closed, accretive operators, weak* compact,homeomorphism, Banach Alaoglu theorem 


\section{Introduction}

The concept of 2- metric spaces, linear 2- normed spaces and 2-inner product spaces, introduced by S. Gahler in 1963, paved the way for a number of authors like, A. White, Y. J. Cho, R. Freese, C. R. Diminnie, for working on possible applications of Metric geometry, Functional Analysis and Topology as a new tool. A systematic presentation of the recent results related to the Geometry of linear 2-normed spaces as well as an extensive list of the related references can be found in the book [1]. In [4] S. Gahler introduced the following definition of linear 2-normed spaces.

\section{Preliminaries}

Definition 2.1 (3). Let $X$ be a real linear space of dimension greater than 1 and $\|.,$.$\| be a real valued function on X \times X$ satisfying the properties,

A1: $\|x, y\|=0$ iff $x$ and $y$ are linearly dependent

A2: $\|x, y\|=\|y, x\|$

A3: $\|\alpha x, y\|=|\alpha|\|y, x\|$

$A 4:\|x+y, z\| \leq\|x, z\|+\|y, z\|$

for every $x, y, z \in X$ and $\alpha \in R$

then the function $\|.,$.$\| is called a 2-norm on X$. The pair $(X,\|.,\|$.$) is$ called a linear 2- normed space.

Some of the basic properties of 2-norms, they are non-negative and $\|x, y+\alpha x\|=\|x, y\|$ for all $x$ and $y$ in $X$ and for every $\alpha$ in $R$.

The most standard example for a linear 2-normed space is $X=R^{2}$ equipped with the following 2-norm,

$$
\left\|x_{1}, x_{2}\right\|=a b s \operatorname{det}\left(\begin{array}{ll}
x_{11} & x_{12} \\
x_{21} & x_{22}
\end{array}\right) \text { where } x_{i}=\left(x_{i 1}, x_{i 2}\right) \text { for } i=1,2
$$

Every linear 2-normed space is a locally convex TVS. In fact, for a fixed $b \in X, P_{b}(x)=\|x, b\|$ is a semi norm, where $x \in X$ and the family $\left\{P_{b} ; b \in X\right\}$ of semi norms generates a locally convex topology on $X$.

Definition 2.2 (3). Let $(X,\|.,\|$.$) be a linear 2-normed space, then a$ map $T: X \times X \rightarrow R$ is called a 2-linear functional on $X$ whenever for every $x_{1}, x_{2}, y_{1}, y_{2} \in X$ and $\alpha, \beta \in R$

(i) $T\left(x_{1}+x_{2}, y_{1}+y_{2}\right)=T\left(x_{1}, y_{1}\right)+T\left(x_{1}, y_{2}\right)+T\left(x_{2}, y_{1}\right)+T\left(x_{2}, y_{2}\right)$

(ii) $T\left(\alpha x_{1}, \beta y_{1}=\alpha \beta T\left(x_{1}, y_{1}\right)\right.$

hold. 
A 2-linear functional $T: X \times X \rightarrow R$ is said to be bounded if there exists a real number $M>0$ such that $|T(x, y)| \leq M\|x, y\|$ for all $x, y$ in $X$.

The norm of the 2-linear functional $T: X \times X \rightarrow R$ is defined for all $x, y$ in $X$ by

$$
\|T\|=\inf \{M>0 ;|T(x, y)| \leq M\|x, y\|\} .
$$

It can be seen that

$$
\begin{aligned}
& \|T\|=\sup \{|T(x, y)| ;\|x, y\| \leq 1\} \\
& \quad=\sup \{|T(x, y)| ;\|x, y\|=1\} \\
& \quad=\sup \left\{\frac{|T(x, y)|}{\|x, y\|} ;\|x, y\| \neq 0\right\}
\end{aligned}
$$

Definition 2.3 (2). Let $(X,\|.,\|$.$) be a linear 2- normed space, E$ be a subset of $X$ then the sequentially closure of $E$ is $\bar{E}=\left\{x \in X: x_{n} \subset\right.$ $\left.E / x_{n} \rightarrow x\right\}$. We say, $E$ is sequentially closed if $E=\bar{E}$.

Definition 2.4 (3). Let $X_{z}^{*}$ be the set of all bounded linear 2- functional on $X \times V\langle z\rangle$ then the duality map is defined by $I(x, z)=\{F \in$ $X_{z}^{*} ; F(x, z)=\|x, z\|^{2}$ and $\left.\|F\|=\|x, z\|\right\}$

\section{Main Results}

Let $(X,\|.,\|$.$) be a linear 2- normed space and A: D(A) \subset X \rightarrow X$ be an operator with domain $D(A)=\{x \in X ; A x \neq 0\}$ and range $R(A)=$ $\cup\{A x ; x \in D(A)\}$. We may identify $\mathrm{A}$ with its graph and the closure of $\mathrm{A}$ with the closure of its graph.

Definition 3.1. : An operator $A: D(A) \subset X \rightarrow X$ is said to be accretive if, for every $z \in D(A)$

$$
\|x-y, z\| \leq\|(x-y)+\lambda(A x-A y), z\| \text { for all } x, y \in D(A) \text { and } \lambda>0 .
$$

Throughout this article $[x, y] \in A$ means $x, y \in X$ such that $y=A x$.

Definition 3.2. : An operator $A: D(A) \subset X \rightarrow X$ is said to be $\mathrm{m}$ accretive if $R(I+\lambda A)=X$ for $\lambda>0$.

An operator $A: D(A) \subset X \rightarrow X$ and $B: D(B) \subset X \rightarrow X$ be two operators then $B$ is said to be an extension of $A$ if $D(A) \subset D(B)$ and $A x=B x$ for every $x \in D(A)$, denote it by $A \subset B$.

Definition 3.3. : An operator $A: D(A) \subset X \rightarrow X$ is said to be a maximal accretive operator in $X$ if $A$ is an accretive operator in $X$ and for every accretive operator $B$ of $X$ with $A \subset B$ then $A=B$. 
Theorem 3.4. If $A$ is an $m$-accretive operator in $X$ then $A$ is a maximal accretive operator.

Proof: Let $\mathrm{B}$ be an accretive operator with $A \subset B$. Let $\lambda>0$ and $[x, y] \in B$.

Since A is m- accretive we have $x+\lambda y \in R(I+\lambda A)$ implies there exists $\left[x_{1}, y_{1}\right] \in A$ such that $x+\lambda y=x_{1}+\lambda y_{1}$

Since $\mathrm{B}$ is accretive and $\left[x_{1}, y_{1}\right] \in B$ we have for every $z \in X$,

$\left\|x-x_{1}, z\right\| \leq\left\|\left(x-x_{1}\right)+\lambda\left(B x-B x_{1}\right), z\right\|$

$=\left\|\left(x-x_{1}\right)+\lambda\left(y-y_{1}\right), z\right\|$

$=\left\|(x+\lambda y)-\left(x_{1}+\lambda y_{1}\right), z\right\|=\|0, z\|$ for every $z \in X$

$=0$

implies $x-x_{1}=0$ and $x=x_{1}$

Therefore $y=y_{1}$ implies $[x, y] \in A$. So $\mathrm{A}=\mathrm{B}$.

Hence $\mathrm{A}$ is a maximal accretive operator.

Lemma 3.5. Let $A$ be an accretive operator in $X$ and let $(u, v) \in X \times X$ then $A$ is maximal accretive in $X$ iff for every $[x, y] \in A$ and $z \in X$ and $\lambda>0$ one has $\|x-u, z\| \leq\|(x-u)+\lambda(y-v), z\|$ implies $[u, v] \in A$.

Proof:

Let $\mathrm{A}$ be a maximal accretive operator in X. Put $T=A \cup[u, v]$

Suppose $\|x-u, z\| \leq\|(x-u)+\lambda(y-v), z\|$ for every $[x, y] \in A, z \in X$ and $\lambda>0$

then $\mathrm{T}$ is accretive in $\mathrm{X}$ and $A \subset T$ implies $[u, v] \in A$

Conversely, suppose that if $\mathrm{A}$ is accretive operator in $\mathrm{X}$ and

$\|x-u, z\| \leq\|(x-u)+\lambda(y-v), z\|$ for every $[x, y] \in A, z \in X$ and $\lambda>0$ implies $[u, v] \in A$

Let $\mathrm{B}$ be accretive in $\mathrm{X}$ with $A \subset B$ and $\left[x_{1}, y_{1}\right] \in B$

Since $\mathrm{B}$ is accretive in $\mathrm{X}$, for every $[x, y] \in A, z \in X$ and $\lambda>0$ one has $\left\|x-x_{1}, z\right\| \leq\left\|\left(x-x_{1}\right)+\lambda\left(B x-B x_{1}\right), z\right\|=\left\|\left(x-x_{1}\right)+\lambda\left(y-y_{1}\right), z\right\|$ which

implies $\left[x_{1}, y_{1}\right] \in A$. Therefore $B \subset A$. So $A=B$.

Hence $\mathrm{A}$ is maximal accretive in $\mathrm{X}$.

Theorem 3.6. If $A$ is an accretive operator in $X$ then there exists a maximal accretive operator containing $A$. 
Proof:

Let $B=\{B$; $\mathrm{B}$ is accretive in $\mathrm{X}$ and $A \subset B\}$ then $(B, \subset)$ is a partially ordered set.

Let $\mathrm{T}$ be a totally ordered set with $T \subset B$ then by Zorn's lemma there exists a maximal element in $\mathrm{B}$, is a maximal accretive operator containing A.

Theorem 3.7. Let $A$ be an accretive operator in $X$ then the closure $\bar{A}$ of $A$ is accretive.

Proof:

Let $\left[x_{1}, y_{1}\right],\left[x_{2}, y_{2}\right] \in \bar{A}$ then there exists sequences $\left\{\left[x_{n}, y_{n}\right]\right\},\left\{\left[x_{m}, y_{m}\right]\right\}$ in A such that $x_{n} \rightarrow x_{1} ; y_{n} \rightarrow y_{1} ; x_{m} \rightarrow x_{2} ; y_{m} \rightarrow y_{2}$ and $\lambda>0$.

Since $\mathrm{A}$ is accretive in $X$ one has

$\left\|x_{n}-x_{m}, z\right\| \leq\left\|\left(x_{n}-x_{m}\right)+\lambda\left(A x_{n}-A x_{m}\right), z\right\|$ for every $z \in X$ $=\left\|\left(x_{n}-x_{m}\right)+\lambda\left(y_{n}-y_{m}\right), z\right\|$ for every $z \in X$

as $n \rightarrow \infty,\left\|x_{1}-x_{2}, z\right\| \leq\left\|\left(x_{1}-x_{2}\right)+\lambda\left(y_{1}-y_{2}\right), z\right\|$ for every $z \in X$

implies $\bar{A}$ is accretive in $\mathrm{X}$.

Theorem 3.8. Let $A$ be a maximal accretive operator in $X$ then $A$ is sequentially closed.

Proof: For all $x_{n}, y_{n} \in D(A)$, Let $\left\{\left[x_{n}, y_{n}\right]\right\}$ in A such that $x_{n} \rightarrow u, y_{n} \rightarrow$ $v$ and $\lambda>0$

Since $\mathrm{A}$ is accretive in $\mathrm{X}$ and $[x, y] \in A$ implies $\left\|x-x_{n}, z\right\| \leq \|(x-$ $\left.x_{n}\right)+\lambda\left(y-y_{n}\right), z \|$ for every $z \in X$

as $n \rightarrow \infty$ we have $\|x-u, z\| \leq\|(x-u)+\lambda(y-v), z\|$ for every $z \in X$

Therefore, by Lemma $3.6[u, v] \in A$. Hence A is sequentially closed.

Corollary 3.9. If $A$ is an m-maximal accretive operator in $X$ then $A$ is sequentially closed.

Proof: We have an m-accretive operator $\mathrm{A}$ in $\mathrm{X}$ is a maximal accretive operator in X. Hence by Theorem 3.8, A is sequentially closed.

Next we prove analogous of Banach Alaoglu theorem in linear 2- normed spaces.

Theorem 3.10. Let $X$ be a linear 2- normed space then the closed unit ball of $X_{z}^{*}$ is weak* compact, i.e. $B=\left\{f \in X_{z}^{*} ;\|f\| \leq 1\right\}$ is compact for the weak ${ }^{*}$ topology. 
Proof:

If $f \in B$ then $|f(x, z)| \leq\|f\|\|x, z\|$ for every $x, z \in X$

Let $D_{x, z}=\{\lambda \in R ;|\lambda| \leq\|x, z\|\}$ be a closed interval then it is compact.

We have $f(x, z) \in D_{x, z}$ for every $x, z \in X$. Take $D=\Pi_{x \in X} D_{x, z}$ for every $z \in X$. Equip product topology on D then, by Tychnoff's theorem D is compact.

Consider the canonical projection $\Pi_{x, z}: D \rightarrow D_{x, z}$

Equip B with the relative topology induced by weak* topology. So it is enough to prove that $\mathrm{B}$ is homeomorphic with a closed subset $\mathrm{C}$ of $\mathrm{D}$.

Define $T: B \rightarrow D$ as follows:

If $f \in B$ then $f(x, z) \in D_{x, z}$ for every $x, z \in X$

So, define $T f=(f(x, z))_{x, z \in X}$ of $\mathrm{D}$ has the property that $(x, z)^{t h}$ coordinate is a 2-linear functional of index $(x, z)$.

Construct the set $\mathrm{C}$ of all $\left(\lambda_{x, z}\right)_{x, z \in X} \in$ in D such that

$\lambda_{\left(x_{1}+x_{2}, z_{1}+z_{2}\right)}=\lambda_{x_{1}, z_{1}}+\lambda_{x_{1}, z_{2}}+\lambda_{x_{2}, z_{1}}+\lambda_{x_{2}, z_{2}}$

$\lambda_{\alpha x_{1}, \beta z_{1}}=\alpha \beta \lambda_{x_{1}, z_{1}}$ for every $x_{1}, x_{2}, z_{1}, z_{2} \in X$ and $\alpha, \beta$ in $R$

We have $T(B) \subset C$

If $\lambda_{x, z} \in C$ for $x, z \in X$

Define $f: X \times X \rightarrow R$ by $f(x, z)=\lambda_{x, z}$ is a 2-linear functional on X.

Also $|f(x, z)|=\left|\lambda_{x, z}\right| \leq\|x, z\|$ implies $\|f\| \leq 1$. Therefore $f \in B$. $C$.

And $T f=f(x, z)_{x, z \in X}=\left(\lambda_{x, z}\right)_{x, z \in X}$. So $C \subset T(B)$. Therefore $T(B)=$

Next we have to prove that,

(i) $\mathrm{T}$ is one-to-one

(ii) $\mathrm{C}$ is a closed subset of $\mathrm{D}$

(iii) $\mathrm{T}$ is bicontinuous (ie; homeomorphiism) from $\mathrm{B}$ onto $T(B)=C$

For,

(i) Let $f, g \in B$ with $T f=T g$ then $f(x, z)=g(x, z)$ for every $x, z \in X$ implies $f=g$. So $\mathrm{T}$ is one-to-one.

(ii) For $x_{1}, x_{2}, z_{1}, z_{2} \in X$, Define $\phi: D \rightarrow R$ by $\phi\left(\lambda_{x, z}\right)=\lambda_{\left(x_{1}+x_{2}, z_{1}+z_{2}\right)}-$ $\lambda_{x_{1}, z_{1}}-\lambda_{x_{1}, z_{2}}-\lambda_{x_{2}, z_{1}}-\lambda_{x_{2}, z_{2}}$

Take $u=\lambda_{x, z}$ then we have $\phi(u)=\pi_{\left(x_{1}+x_{2}, z_{1}+z_{2}\right)}(u)-\pi_{x_{1}, z_{1}}(u)-$ $\pi_{x_{1}, z_{2}}(u)-\pi_{x_{2}, z_{1}}(u)-\pi_{x_{2}, z_{2}}(u)$

Since $\pi$ is continuous we have $\phi$ is continuous.

Define $\phi^{-1}[0]=\left\{\lambda_{x, z} \in D: \lambda_{\left(x_{1}+x_{2}, z_{1}+z_{2}\right)}=\lambda_{x_{1}, z_{1}}+\lambda_{x_{1}, z_{2}}+\lambda_{x_{2}, z_{1}}+\right.$ $\left.\lambda_{x_{2}, z_{2}}\right\}$. Then $\phi^{-1}[0]$ is closed in D. Denote this closed set by $C_{\left(x_{1}, x_{2}, z_{1}, z_{2}\right)}$.

Similarly, for fixed $v_{1}, v_{2} \in X$ and $\alpha, \beta \in R$ the set $\left\{\left(\lambda_{x, z}\right)_{x, z \in X} ; \lambda_{\alpha x_{1}, \beta z_{1}}=\right.$ $\left.\alpha \beta \lambda_{x_{1}, z_{1}}\right\}$ is closed in D. Denote it by $C_{\left(v_{1}, v_{2}, \alpha, \beta\right)}$. 
Therefore, $C=\left(\cap C_{\left(x_{1}, x_{2}, z_{1}, z_{2}\right)}\right) \cap\left(\cap C_{\left(v_{1}, v_{2}, \alpha, \beta\right)}\right)$ where $x_{1}, x_{2}, z_{1}, z_{2}, v_{1}, v_{2}$ varies over $\mathrm{X}$ and $\alpha, \beta$ varies over $\mathrm{R}$. Hence $\mathrm{C}$ is closed in $\mathrm{D}$.

(iii) In the view of (i) $\mathrm{T}$ maps bijectively onto $T(B)=C$

Consider a sub basic neighbourhood of $f_{0}$ for the relative weak ${ }^{*}$ topology on $\mathrm{B}$ of the form:

Let $e \in X$ then $V=\left\{f \in B ;\left\|f\left(x_{0}, y_{0}\right)-f_{0}\left(x_{0}, y_{0}\right), e\right\| \leq \varepsilon\right\}$

Therefore, $T(V)=\left\{[f(x, y)]_{x, z} \in X ; f \in V\right\}$

$$
\begin{aligned}
& \quad=\left\{[f(x, y)]_{x, z \in X} ; f \in B \text { with }\left\|f\left(x_{0}, y_{0}\right)-f_{0}\left(x_{0}, y_{0}\right), e\right\| \leq \varepsilon\right\} \\
& =\left\{[f(x, y)]_{x, z \in X} ; f \in B \text { with }\left\|\pi_{\left(x_{0}, y_{0}\right)}(T f)-\pi_{\left(x_{0}, y_{0}\right)}\left(T f_{0}\right), e\right\| \leq \varepsilon\right\}
\end{aligned}
$$

is a sub basic neighbourhood of $T f_{0}$ for the relative topology induced on $T(B)=C$ by the product topology on D. So,T is bicontinuous from B onto $T(B)=C$

Theorem 3.11. Let $X$ be a linear 2-normed space and $x, y \in X$ then for every $z \in X,\|x, z\| \leq\|x+\alpha y, z\|$ for every $\alpha>0$ iff there is $F \in I(x, z)$ such that $\operatorname{Re}((y, z), F) \geq 0$ ["Re" means "real part of"]

Proof:

If $\mathrm{x}=0$ then the result holds true.

Assume that $x \neq 0$. Suppose $\operatorname{Re}((y, z), F) \geq 0$ for some $F \in I(x, z)$ then $\alpha>0$

$\|x, z\|^{2}=F(x, z)=\operatorname{Re}(F(x, z)) \leq \operatorname{Re}(F(x+\alpha y)) \leq\|F\|\|x+\alpha y, z\|$ for

Since, $\|F\|=\|x, z\|$ we have $\|x, z\| \leq\|x+\alpha y, z\|$ for $\alpha>0$

Conversely, suppose that $\|x, z\| \leq\|x+\alpha y, z\|$ for $\alpha>0$

For each $\alpha>0$ let $F_{\alpha} \in I(x+\alpha y, z)$ and $g_{\alpha}=\frac{F_{\alpha}}{\left\|F_{\alpha}\right\|}$ then $\left\|g_{\alpha}\right\|=1$

Then,

$\|x, z\| \leq\|x+\alpha y, z\|=g_{\alpha}(x+\alpha y, z)=\operatorname{Re}\left[g_{\alpha}(x, z)\right]+\alpha \operatorname{Re}\left[g_{\alpha}(y, z)\right] \leq$ $\|x, z\|+\alpha \operatorname{Re}\left[g_{\alpha}(y, z)\right]$

implies $\lim \left\{i n f_{(\alpha \downarrow 0)} \operatorname{Re}\left[(x, z), g_{\alpha}\right]\right\} \geq\|x, z\|$ and $\operatorname{Re}\left[(y, z), g_{\alpha}\right] \geq 0$

By the above theorem, the closed unit ball of $X_{z}^{*}$ is weak* compact then the net $\left\{g_{\alpha}\right\}$ has a cluster point $g$ with $\|g\|=1$.

$\operatorname{Re}\left[(x, z), g_{\alpha} \geq\|x, z\|\right.$ and $\operatorname{Re}\left[(y, z), g_{\alpha}\right] \geq 0$ implies $\operatorname{Re}\left[\frac{(x, z)}{\|x, z\|}, g_{\alpha}\right] \geq 1$

implies $\|g\|=1$ and $g(x, z)=\|x, z\|$

Take $F=\|x, z\| g$ then $F(x, z)=\|x, z\| g(x, z)=\|x, z\|^{2}$. Therefore, $F \in I(x, z)$ and $\operatorname{Re}[(y, z), F] \geq 0$

Remark 3.12. From the above theorem we get " $A$ is an accretive operator in a linear 2-normed space $X$ iff for every $u, v \in D(A)$ there exists $f \in I(u-v, z)$ such that $\operatorname{Re}[f(A u-A v, z)] \geq 0 "$. 


\section{References}

[1] Berbarian, Lectures in Operator theory, Springer, 1973.

[2] Fatemeh Lael and Kourosh Nourouzi, Compact Operators Defined on 2-Normed and 2-Probabilistic Normed Spaces, Hindawi Publishing Corporation,Mathematical Problems in Engineering, Volume 2009 (2009), Article ID 950234, 17 pages.

[3] Raymond W. Freese,Yeol Je Cho, Geometry of linear 2-normed spaces, Nova Science publishers, Inc, Newyork, (2001).

[4] Shih â" sen Chang, Yeol Je Cho, Shin Min Kang, Nonlinear operator theory in Probabilistic Metric spaces, Nova Science publishers, Inc, Newyork, (2001).

[5] S. Gahler, Siegfried 2-metrische Raume und ihre topologische struktur, Math. Natchr. 26(1963),115-148 .

[6] T. Kato, Nonlinear semigroups and evolution equations, J. Math. Soc. Japan, Vol. 19, No. 4, (1967).

\section{P. K. Harikrishnan}

Department of Mathematics

Manipal Institute of Technology

Manipal, Karnataka

India

e-mail : pkharikrishnans@gmail.com

\section{Bernardo Lafuerza Guillén'}

Department of Statistics and Applied Mathematics

University of Almería

Almería

Spain

e-mail : blafuerz@ual.es

and 
K. T. Ravindran

P G Department and Research Centre in Mathematics

Payyanur College

Payyanur, Kerala

India

e-mail : drktravindran@gmail.com 